\title{
The effect of triglyceride chain length combined with exercise on appetite, satiety and energy balance
}

\author{
T.J. Maher, A. El-Chab and M.E. Clegg \\ Oxford Brookes Centre for Nutrition and Health, Faculty of Health and Life Sciences, Oxford Brookes University, \\ $O X 3 O B P$.
}

Medium chain triglycerides (MCT) have been shown to increase energy expenditure ${ }^{(1,2)}$ as well as increase satiety and suppress food intake ${ }^{(3-5)}$. The effects of exercise on appetite regulation are equivocal, but it is well documented that acute exercise does lead to energy deficits ${ }^{(6)}$. These effects are achieved through separate mechanisms, and thus there is potential for the combination of MCT and exercise to yield increased satiety. Therefore, the aim of this study was to elucidate the effects of MCT, exercise, or a combination of the two on subjective appetite sensations, energy intake and overall energy balance.

Twelve healthy males $\left(27 \pm 11.43\right.$ years, BMI: $\left.23.76 \pm 2.85 \mathrm{~kg} / \mathrm{m}^{2}\right)$ completed four trials in random order. After a $24 \mathrm{~h}$ standardisation period and a $12 \mathrm{~h}$ fast, participants consumed a porridge breakfast which contained $165 \mathrm{kcal}$ of either vegetable oil or MCT oil. Participants consumed each breakfast on two separate occasions; one followed by complete rest for $4 \mathrm{~h}$, one followed by rest broken up with an hour of cycling at $65 \% \mathrm{VO}_{2 \text { peak }}$ at $3 \mathrm{~h}$. Expired air samples (for calculation of energy expenditure) and subjective ratings of appetite, on visual analogue scales (VAS), were taken every $30 \mathrm{~min}$ for 4 hours. At $4 \mathrm{~h}$, participants consumed a multi-item buffet lunch ad libitum to satiation, after which they completed diet diaries for the rest of the day.

There was no effect of either lipid or exercise condition on energy intake at the ad libitum meal (Control-Rest $6278.4 \pm 1758.62 \mathrm{~kJ}$; Control-Exercise $6785.2 \pm 1370.5 \mathrm{~kJ}$; MCT-Rest $6077.1 \pm 1853.5 \mathrm{~kJ}$; MCT-Exercise $6794.4 \pm 2030.3 \mathrm{~kJ} ; P \geq 0.05)$. There were also no differences for any of the VAS scores (all $P \geq 0.05)$ and no effect on ratings of nausea $(P \geq 0.05)$. There were significant main effects for breakfast $(P=0.031)$ and exercise condition $(P<0.001)$ on total energy expenditure. Consumption of the MCT breakfast led to greater energy expenditure compared to the control breakfast, and the exercise trials led to greater energy expenditure than the resting trials (Control-Rest 198.2 $\pm 138.2 \mathrm{~kJ}$; Control-Exercise 3045.8 $\pm 606.2 \mathrm{~kJ}$; MCT-Rest 211.1 \pm 186.6 kJ; MCT-Exercise 3272.4 \pm 763.2 $\mathrm{kJ})$, but there was no significant interaction between breakfast and condition $(P \geq 0.05)$.

The results of this study indicate that MCT can increase dietary induced thermogenesis, although had no impact on satiety. There is also no cumulative effect of combining MCT with exercise on either suppressing energy intake or increasing energy expenditure.

1. Clegg M, Golsorkhi M \& Henry C (2013). Eur J Nut 52, 1579-85

2. Ogawa A, Nosaka N, Kasai M et al. (2007). J Oleo Sci 56, 283-7

3. Kinsella R, Maher T \& Clegg M (2017). Physiol Behav

4. Van Wymelbeke V, Himaya A, Louis-Sylvestre J et al. (1998). Am J Clin Nutr 68, 226-34

5. Rolls B, Gnizak N, Summerfelt A et al. (1988). Am J Clin Nutr 48, 66-71

6. Schubert M, Desbrow B, Sabapathy S. et al. (2013). Appetite 63, 92-104. 